\title{
THE CHARACTERISTIC NUMBERS OF 4-DIMENSIONAL KÄHLER MANIFOLDS
}

\author{
Y. L. XIN \\ Dedicated to the author's teacher Professor Buchin Su
}

\section{Introduction}

There have been many results about the relation between the curvature of a Riemannian manifold $M$ and its characteristic numbers. S. S. Chern and J. Milnor [3] proved that a 4-dimensional manifold with sectional curvature everywhere of the same sign has nonnegative Euler number. M. Berger [1] and N. Hitchin [6] considered the case of an Einstein manifold. H. Donnelly [4] obtained inequalities involving the Euler number and the Pontrjagin number of Einstein Kähler manifolds. S. T. Yau [11] and A. Polombo [8] generalized Gray-Hitchin-Thorpe [5], [6], [9] inequality to $k$-Ricci pinched manifolds and considered the $k$-sectionally pinched case.

In the present paper the similar problem for $k$-Ricci pinched Kähler manifold is considered, and a generalization of Donnelly's inequalities is obtained (Theorem 1).

On the other hand R. Bishop and S. I. Goldberg [2] proved that a 4-dimensional Kähler manifold with holomorphic sectional curvature everywhere of the same sign has nonnegative Euler number. This result is improved in Theorem 2 of this paper.

Thus the main results are the following two theorems.

Theorem 1. Let $M$ be a compact oriented 4-dimensional Kähler manifold with Euler number $\chi$ and Pontrjagin number $p$. If $M$ is $k$-Ricci pinched with $k \geqslant \sqrt{2} / 2$, then the inequalities

$$
\chi+\frac{3-5 k^{2}}{2 k^{2}} p \geqslant 0,
$$

Received, November 1, 1979, and, in revised form, April 14, 1980. The author would like to thank Professors C. N. Yang, C. H. Gu and A. Phillips for their help, and is grateful to the State University of New York at Stony Brook and University of California at Berkeley for their hospitality. 


$$
\chi+\frac{1}{2} p \geqslant 0
$$

are valid. Furthermore, if the equality in (1) occurs, then $M$ must be in one of the following three cases:

(i) $M$ has constant holomorphic curvature,

(ii) the universal covering manifold of $M$ is a $K_{3}$ surface,

(iii) $M$ is flat.

If the equality in (2) occurs, then $M$ must be in one of cases (ii) and (iii) above.

Theorem 2. Let $M$ be a compact oriented 4-dimensional Kähler manifold with Euler number $\chi$ and Pontrjagin number $p$. If $M$ is $\lambda$-holomorphically pinched with $\lambda \geqslant 0$, then

$\chi+\frac{1}{2} p \geqslant 0, \quad \chi+\min \left(\frac{1-2 \lambda-5 \lambda^{2}}{6 \lambda^{2}}, \frac{\lambda^{2}}{\lambda^{2}-4}\right) p \geqslant 0$ for $\frac{1}{4} \leqslant \lambda \leqslant 1$,

and, otherwise

$$
\chi+\frac{\lambda^{2}}{1-4 \lambda^{2}} p \geqslant 0, \quad \chi+\frac{\lambda^{2}}{\lambda^{2}-4} p \geqslant 0 .
$$

We should point out that A. Polombo [7] has obtained similar results, which however do not cover the above theorems.

\section{Preliminary notation}

First of all, we construct a special Hermitian basis at any point $p$ in a 4-dimensional Kähler manifold $M$. Let $e_{1}$ and $e_{2}$ be unit eigenvectors of the Ricci curvature such that it reaches its maximum and minimum respectively. It is clear that $e_{1}$ and $e_{2}$ are mutually perpendicular. Therefore using the canonical almost complex structure $J$ we obtain a Hermitian basis $\left\{e_{1}, J e_{1}, e_{2}, J e_{2}\right\}$ which diagonalizes the Ricci curvature tensor. In this case $R_{11}=R_{22}$ and $R_{33}=R_{44}$.

From the author's previous paper [10], we have the Euler number $\chi$ and the Pontrjagin number $p$ for any 4-dimensional Kähler manifold:

$$
\begin{gathered}
\chi=\frac{1}{8 \pi^{2}} \int_{M}\left(\left|W^{-}\right|^{2}+\frac{S^{2}}{12}-2 \mid R^{+-\left.\right|^{2}}\right) \Omega, \\
p=\frac{1}{4 \pi^{2}} \int_{M}\left(\frac{S^{2}}{24}-\left|W^{-}\right|^{2}\right) \Omega,
\end{gathered}
$$

where $W^{-}$is the antiself dual part of the conformal curvature tensor, $R^{+-}$is the part of the Riemannian curvature tensor which is self dual on the first two indices as well as antiself dual on the last two [10], $S$ is the scalar curvature, and $\Omega$ is the volume form of the manifold. 
Equivalently, (4) and (5) can be expressed in another form:

$$
\begin{aligned}
& \chi=\frac{1}{8 \pi^{2}} \int_{M}\left(|R-|^{2}+\frac{1}{16} S^{2}-2 \mid R^{+-\left.\right|^{2}}\right) \Omega, \\
& p=\frac{1}{4 \pi^{2}} \int_{M}\left(\frac{S^{2}}{16}-|R-|^{2}\right) \Omega,
\end{aligned}
$$

where $R^{--}$is the part of the Riemannian curvature tensor and is antiself dual on both pairs of indices.

By directly computing, we have

$$
\begin{gathered}
\mid R^{+-\left.\right|^{2}}=\frac{1}{4}\left(R_{1212}-R_{3434}\right)^{2}, \\
\left|R^{+-}\right|^{2}=\frac{1}{16}\left(R_{11}+R_{22}-R_{33}-R_{44}\right)^{2}=\frac{1}{4}\left(R_{11}-R_{33}\right)^{2}
\end{gathered}
$$

under the special Hermitian basis $\left\{e_{1}, J e_{1}, e_{2}, J e_{2}\right\}$.

Let $X$ and $Y$ be perpendicular unit tangent vectors of $M$ at any point $p$, such that $\langle X, J Y\rangle=0$. Then we have the formula [2]

$$
\begin{aligned}
K(X, Y)+K(X, J Y)=\frac{1}{4}[H(X+J Y) & +H(X-J Y)+H(X+Y) \\
& +H(X-Y)-H(X)-H(Y)]
\end{aligned}
$$

where $K(X, Y)$ is the sectional curvature of the plane spanned by $X, Y$, and $H(X)=K(X, J X)$. By (7) we obtain the components of the Ricci curvature tensor:

$$
\begin{aligned}
R_{11}=K\left(e_{1}, J e_{1}\right)+ & K\left(e_{1}, e_{2}\right)+K\left(e_{1}, J e_{2}\right) \\
=H\left(e_{1}\right)+\frac{1}{4}[ & H\left(e_{1}+J e_{2}\right)+H\left(e_{1}-J e_{2}\right)+H\left(e_{1}+e_{2}\right) \\
& \left.+H\left(e_{1}-e_{2}\right)-H\left(e_{1}\right)-H\left(e_{2}\right)\right], \\
R_{33}=H\left(e_{2}\right)+\frac{1}{4}[ & H\left(e_{1}+J e_{2}\right)+H\left(e_{1}-J e_{2}\right)+H\left(e_{1}+e_{2}\right) \\
& \left.+H\left(e_{1}-e_{2}\right)-H\left(e_{1}\right)+H\left(e_{2}\right)\right],
\end{aligned}
$$

from which it follows that

$$
\begin{aligned}
S= & H\left(e_{1}+J e_{2}\right)+H\left(e_{1}-J e_{2}\right)+H\left(e_{1}+e_{2}\right) \\
& +H\left(e_{1}-e_{2}\right)+H\left(e_{1}\right)+H\left(e_{2}\right),
\end{aligned}
$$

and (6) can be written in the following form:

$$
\left|R^{+-}\right|^{2}=\frac{1}{4}\left[H\left(e_{1}\right)-H\left(e_{2}\right)\right]^{2} .
$$


By definition a $k$-Ricci pinched manifold is one in which there is a number $k>0$ such that

$$
\frac{1}{4}|S| \geqslant k\left|R_{i i}\right|
$$

for all $i$. It is easy to see $k \leqslant 1$. If the equality in (10) occurs, then either $k=1$ or $S=0$. Both conditions imply that the manifold is an Einstein manifold; furthermore in the second case it must be Ricci flat.

\section{Proof of Theorem 1}

From the pinching condition (10), we have

$$
R_{11}^{2}+R_{33}^{2} \leqslant \frac{S^{2}}{8 k^{2}}
$$

Substituting the above inequality into $\left(6^{\prime}\right)$ yields the following:

$$
\begin{aligned}
\left|R^{+-}\right|^{2} & =\frac{1}{4}\left(R_{11}-R_{33}\right)^{2}=\frac{1}{2}\left(R_{11}^{2}+R_{33}^{2}\right)-\frac{1}{4}\left(R_{11}+R_{33}\right)^{2} \\
& \leqslant \frac{S^{2}}{16 K^{2}}-\frac{S^{2}}{16}=\frac{1-K^{2}}{16 K^{2}} S^{2} .
\end{aligned}
$$

If the equality holds above, then the equality also holds in (10) for $k$-Ricci pinched manifolds. Thus the equality in (11) occurs iff $k=1$ or $S=0$.

From (4), (5) and (11), we have

$$
\chi+b p \geqslant \frac{1}{8 \pi^{2}} \int_{M}\left[(1-2 b)\left|W^{-}\right|^{2}+\frac{5 k^{2}-3+2 b k^{2}}{24 k^{2}} S^{2}\right] \Omega
$$

for any real $b$. Taking $b=\frac{1}{2}\left(3-5 k^{2}\right) / k^{2}$, we reduce (12) to

$$
\chi+\frac{3-5 k^{2}}{2 k^{2}} p \geqslant \frac{3}{8 \pi^{2}} \int_{M} \frac{2 k^{2}-1}{k^{2}}\left|W^{-}\right|^{2} \Omega,
$$

which gives (1) when $K \geqslant \sqrt{2} / 2$. The equality in (1) occurs only if one of the following conditions holds:

(i) $K=1,\left|W^{-}\right|=0$ and $S \neq 0$;

(ii) $S=0, k^{2}=\frac{1}{2}$ and $\left|W^{-}\right| \neq 0$;

(iii) $S=0,\left|W^{-}\right|=0$.

Under the first condition $M$ has constant holomorphic curvature [10]. The second condition means that the universal covering of $M$ is a $K_{3}$ surface [6]. When $S=0$ and $\left|W^{-}\right|^{2}=0$, then $\chi=0$, which forces $M$ to be flat [1]. Taking $b=\frac{1}{2}$, from (12) we have (2) provided $k \geqslant \sqrt{2} / 2$. If the equality holds in (2), then $M$ satisfies either (ii) or (iii) above. The same discussion as above would not be repeated. 


\section{Proof of Theorem 2.}

If $M$ is a $\lambda$-holomorphically pinched Kähler manifold with $\lambda>0$, then there is a constant $A>0$ such that

$$
\lambda A \leqslant H(X) \leqslant A,
$$

for any $X \in T_{p}(M)$.

The pinching condition (13) and (8) give the inequality

$$
6 \lambda A \leqslant S \leqslant 6 A \text {. }
$$

From (9) and (13) we have

$$
\left|R^{+-}\right|^{2} \leqslant \frac{1}{4}(1-\lambda)^{2} A^{2} .
$$

For any $b \geqslant-1,(4),(5)$ and (15) give

$$
\chi+b p \geqslant \frac{1}{8 \pi^{2}} \int_{M}\left\{(1-2 b)\left|W^{-}\right|^{2}+\left[\left(\frac{5}{2} \lambda^{2}+\lambda-\frac{1}{2}\right)+3 b \lambda^{2}\right] A^{2}\right\} \Omega
$$

Taking $b=\frac{1}{2}$ in (16), we have

$$
\chi+\frac{1}{2} p \geqslant \frac{1}{8 \pi^{2}} \int_{M}\left(4 \lambda^{2}+\lambda-\frac{1}{2}\right) A^{2} \Omega .
$$

Thus

$$
\chi+\frac{1}{2} p \geqslant 0, \quad \text { for } \frac{1}{4} \leqslant \lambda \leqslant 1 .
$$

Taking $b=\frac{1}{6}\left(1-2 \lambda-5 \lambda^{2}\right) / \lambda^{2}$ in (16), we have

$$
x+\frac{1-2 \lambda-5 \lambda^{2}}{6 \lambda^{2}} p \geqslant 0
$$

when $\frac{1}{4} \leqslant \lambda \leqslant 1$.

If we denote

$$
\begin{aligned}
& \varepsilon_{1}^{-}=e_{1} \wedge J e_{1}-e_{2} \wedge J e_{2}, \\
& \varepsilon_{2}^{-}=e_{1} \wedge e_{2}-J e_{2} \wedge J e_{1}, \\
& \varepsilon_{3}^{-}=e_{1} \wedge J e_{2}-J e_{1} \wedge e_{2},
\end{aligned}
$$

then

$$
\begin{aligned}
\left\langle R_{e_{1}^{-}}, \varepsilon_{1}^{-}\right\rangle & =\left\langle R_{e_{1}^{-}}\left(e_{1}\right), J e_{1}\right\rangle-\left\langle R_{e_{1}^{-}}\left(e_{2}\right), J e_{2}\right\rangle \\
& =-\frac{S}{2}+2\left\langle R_{e_{1} J e_{1}}\left(e_{1}\right), J e_{1}\right\rangle+2\left\langle R_{e_{2} J e_{2}}\left(e_{2}\right), J e_{2}\right\rangle \\
& =-\frac{S}{2}+2 H\left(e_{1}\right)+2 H\left(e_{2}\right),
\end{aligned}
$$


from which it follows that

$$
\left|R^{--}\right|^{2}=\left(H\left(e_{1}\right)+H\left(e_{2}\right)-\frac{S}{4}\right)^{2}+L^{2}
$$

where $L^{2}$ is the sum of the squares of all the other entries of the matrix $\left(R^{--}\right)$.

For any $b$ from (9) we have

$$
\begin{gathered}
(1-2 b)\left(H\left(e_{1}\right)+H\left(e_{2}\right)-\frac{1}{4} S\right)^{2}+\frac{1+2 b}{16} S^{2}-2\left|R^{+-}\right|^{2} \\
=(1-2 b)\left(H\left(e_{1}\right)+H\left(e_{2}\right)-\frac{1}{4} S\right)^{2}+\frac{1+2 b}{16} S^{2}-\frac{1}{2}\left(H\left(e_{1}\right)-H\left(e_{2}\right)\right)^{2} \\
(20) \quad\left(\frac{1}{2}-2 b\right)\left[H\left(e_{1}\right)+H\left(e_{2}\right)-\frac{1}{2} S\right]^{2}+\frac{b}{2}\left[S-H\left(e_{1}\right)-H\left(e_{2}\right)\right]^{2} \\
\quad-\frac{b}{2}\left[H\left(e_{1}\right)+H\left(e_{2}\right)\right]^{2}+2 H\left(e_{1}\right) H\left(e_{2}\right) .
\end{gathered}
$$

For $0 \leqslant b \leqslant \frac{1}{4}$ it follows from (13), (14) and (20) that

$$
\begin{aligned}
(1-2 b) & \left(H\left(e_{1}\right)+H\left(e_{2}\right)-\frac{1}{4} S\right)^{2}+\frac{1+2 b}{16} S^{2}-2\left|R^{+-}\right|^{2} \\
& \geqslant\left(8 \lambda^{2} b-2 b+2 \lambda^{2}\right) A^{2}=2\left(\left(4 \lambda^{2}-1\right) b+2 \lambda^{2}\right) A^{2} .
\end{aligned}
$$

For $0 \leqslant b \leqslant \frac{1}{4},\left(4^{\prime}\right),\left(5^{\prime}\right),(19)$ and $(21)$ give

$$
\chi+b p \geqslant \frac{1}{8 \pi^{2}} \int_{M}\left\{(1-2 b) L^{2}+2\left[\left(4 \lambda^{2}-1\right) b+\lambda^{2}\right] A^{2}\right\} \Omega .
$$

Taking $b=\lambda^{2} /\left(1-4 \lambda^{2}\right)$ in (22) yields

$$
\chi+\frac{\lambda^{2}}{1-4 \lambda^{2}} p \geqslant \frac{1}{8 \pi^{2}} \int_{M} \frac{1-6 \lambda^{2}}{1-4 \lambda^{2}} L^{2} \Omega .
$$

Note that $0 \leqslant b \leqslant 1 / 4$. Thus if $\lambda \leqslant \sqrt{2} / 4$, then

$$
\chi+\frac{\lambda^{2}}{1-4 \lambda^{2}} p \geqslant 0 \text {. }
$$

We consider again the case $b \leqslant 0$ in (20). In this case

$$
\begin{array}{r}
(1-2 b)\left(H\left(e_{1}\right)+H\left(e_{2}\right)-\frac{1}{4} S\right)^{2}+\frac{(1+2 b)}{16} S^{2}-2\left|R^{+-}\right|^{2} \\
\geqslant\left(8 b-2 b \lambda^{2}+2 \lambda^{2}\right) A^{2}=\left[\left(8-2 \lambda^{2}\right) b+2 \lambda^{2}\right] A^{2}
\end{array}
$$

from which for any $\lambda \geqslant 0$ we obtain the inequality

$$
\chi+\frac{\lambda^{2}}{\lambda^{2}-4} p \geqslant \frac{1}{8 \pi^{2}} \int_{M} \frac{4+\lambda^{2}}{4-\lambda^{2}} L^{2} \Omega \geqslant 0 .
$$

Therefore inequalities (3) follow from (17), (18), (23) and (24). 
Remarks. 1. A result similar to Theorem 2 holds also in the case of nonpositive holomorphic curvature, but the pinching condition $-A \leqslant H(X)$ $\leqslant \lambda A$ with $\lambda \leqslant 0$ must be substituted for $\lambda A \leqslant H(X) \leqslant A$ with $\lambda \geqslant 0$. It is easy to see that the proof is similar.

2. From (4'), (19) and (20) it follows

$$
\chi=\frac{1}{8 \pi^{2}} \int_{M}\left[L^{2}+\frac{1}{2}\left(H\left(e_{1}\right)+H\left(e_{2}\right)-\frac{1}{2} S\right)^{2}+2 H\left(e_{1}\right) H\left(e_{2}\right)\right] \Omega,
$$

which is nonnegative when holomorphic curvature has the same sign everywhere. This is the theorem of R. Bishop and S. I. Goldberg, which is a special case of Theorem 2 in this paper. It is easy to see that $\chi=0$ forces $M$ to be flat.

\section{References}

[1] M Berger, Sur les variétés d'Einstein compactes, C. R. III ${ }^{e}$ Réunion Math. Expression Latine, Namur (1965) 35-55.

[2] R. L. Bishop \& S. I. Goldberg, Some implications of the generalized Gauss-Bonnet theorem, Trans. Amer. Math. Soc. 112 (1964) 508-535.

[3] S. S. Chern, On curvature and characteristic class of a Riemannian manifold, Abh. Math. Sem. Univ. Hamburg 20 (1955) 117-126.

[4] H. Donnelly, Topology and Einstein Kaehler metrics, J. Differential Geometry 11 (1976) 259-264.

[5] A. Gray, Invariants of curvature operators of four-dimensional Riemannian manifolds, Proc. 13th Biennal Sem. Canad. Math. Congress, Vol. 2, 1971, 42-65.

[6] N. Hitchin, Compact four-dimensional Einstein manifolds, J. Differential Geometry 9 (1974) 435-441.

[7] Albert Polombo, Nombres caractéristiques d'une surface Kählérienne compacte, C. R. Acad. Sc. Paris Sér. A 283 (1976) 1025-1028.

[8] __ Nombres caracteristiques d'une variété Riemannienne de-dimension 4, J. Differential Geometry 13 (1978) 145-162.

[9] J. A. Thorpe, Some remarks on the Gauss-Bonnet integral, J. Math. Mech. 18 (1969) 779-786.

[10] Y. L. Xin, Remarks on characteristic classes of 4-dimensional Einstein manifolds, J. Mathematical Phys. 21 (1980) 343-346.

[11] S. T. Yau, Curvature restrictions on four-manifolds, preprint.

State University of New York, Stony Brook FudAN UNIVERSITY, SHANGHAI 
